\title{
Research Posters
}

R501

\section{Upregulation of $\beta$-Catenin in External Auditory Canal Cholesteatoma}

Ramin Naim, MD (presenter); Clemens Anders, MS; Frank Riedel, MD; Haneen Sadick MD; Karl Hoermann, MD

Mannheim Germany; Mannheim Germany; Mannheim Germany; Mannheim Germany; Mannheim Germany

Problem: The EACC is a rare disease with hyperproliferation and destructive growth into the adjacent structures. Downregulation of $\beta$-catenin (key component of the zonula adherens) is a pivotal factor for loose tissue integrity and invasiveness. TGF- $\beta 1$ was reported to decrease $\beta$-catenin in mammary epithelium. Here, we investigate the abrogation of TGF- $\beta 1$ and $\beta$-catenin expression in EACC culture cells.

Methods: Cultured EACC-specimens were incubated with 6 $\mu \mathrm{mol}$ TGF- $\beta 1$ antisense. After 48 hours, expression of $\beta$-catenin was determined by means of immunohistochemistry.

Results: The cells showed an increased mural reactivity to $\beta$-catenin. Intracellular reactivity was unchanged. The untreated cells showed loss of $\beta$-catenin expression at the membranes.

Conclusion: The predominant membranous location after treatment with TGF- $\beta 1$ antisense suggests increased tendency of the cells for tissue formation and strong cell-cell adhesion rather than migratory and invasive character, thus, TGF- $\beta 1$ antisense application as a useful therapeutical strategy.

Significance: Abrogation of TGF- $\beta 1$ as a useful therapeutical strategy for the treatment of EACC.

R502

\section{Curvature of in Vivo Human Tympanic Membrane}

Jen-Fang Yu, PhD (presenter); Chung-Yi Lee, MD; Jyh-Horng Chen, PhD; Hsin-Chung Lien, PhD; Ta-Te Lin, PhD

Taipei Taiwan (Republic of China); Taipei Taiwan (Republic of China); Taipei Taiwan (Republic of China); Taipei Taiwan (Republic of China); Taipei Taiwan (Republic of China)

Problem: To non-invasively measure the curvature of the in-vivo human tympanic membrane structure for further explaining some of the variability in long-term hearing improvement after tympanoplasty surgery for chronic otitis media.

Methods: All MR images of 5 female and 5 male volunteers $i_{-}$TM, semicircular canals and cochlea have been acquired by a 3-T MR system (Brucker S630) using a surface coil of $9-\mathrm{cm}$ diameter and aged 20 to 35 years. The pixel resolution of the acquired image for the volunteers $i_{-}$right ears were $0.59 \mathrm{~mm}_{i} \mathrm{~N}, 0.63 \mathrm{~mm}_{i} \mathrm{~N}, 0.47 \mathrm{~mm}$ by 3D SNAP sequence and $0.25 \mathrm{~mm}_{i} \mathrm{~N}, 0.25 \mathrm{~mm} ; \mathrm{N}, 2.2 \mathrm{~mm}$ by Multi-slicesingle-echo sequence, respectively. The edge of TM could be detected by the threshold image reconstruction. The radius of curvature of TM was than estimated manually. The curvature could be obtained by the reciprocal of the radius of curvature.

Results: The angle in the apex of the curved cone was 112 degrees. The radius of curvature was $33.8 \mathrm{~mm}$ and the curvature was then $0.0295 / \mathrm{mm}$ for TM structure of the volunteer. The three-dimensional curvature and the variability of dimensional characteristics of the other volunteers $i_{-}$TM were further analyzed. The number of volunteer did not reach a statistically significant level.

Conclusion: The radius of curvature and the curvature of in-vivo human TM were both qualified by the MR image. Using the proposed measurement method, the spatial configuration of the in-vivo TM can be tracked in pre- and postoperation of tympanoplasty non-invasively.

Significance: Knowledge of the structure of the TM may help determine the cause of the continued problems such as the types I, II and III tymanoplasty, age-related changes in the mechanics of the TM can explain part of the age-related hearing loss observed, and some patients with surgically repaired tympanic membrane perforation still have hearing loss.

\section{R503}

\section{Suitability of Formalin-Fixed Acellular Dermis for Tympanic Membrane Repair in Chinchillas \\ Charles Mixson, MD (presenter); Alan J Johnson, MD Augusta $G A$; Augusta $G A$}

Problem: Though an efficacious technique, fasciaform tympanoplasty, as described by Perkins, has a lengthy graft preparation process. The possibility of an endless supply of pre-formed grafts would shorten operative time and lessen donor site morbidity. This paper evaluates the suitability of formalin-fixed Alloderm as a tympanic membrane graft in the chinchilla model.

Methods: Two-mm central perforations were created bilaterally in 15 adult chinchillas. Each chinchilla had a formalin-fixed Alloderm repair in one ear (FF) and an unfixed Alloderm repair (NF) in the other ear as a control (side randomly assigned). The animals were sacrificed after 6 weeks of observation. The tympanic membranes (TM) were otomicroscopically examined for success of repair by a blinded observer. The TM's were then harvested and examined histopathologically by a blinded veterinary pathologist, who assigned a scale of 1 to 5 for inflammation, fibrosis, and cellular ingrowth.

Results: Thirteen chinchillas survived to the evaluation endpoint. The TM's were completely healed in 11 of 13 $(85 \%)$ in each group. Effusions were present in $15 \%$ of NF's 